\title{
Tendiendo puentes: la utilidad de la historia de la ciencia para comprender el proceso de investigación y desarrollo de medicamentos
}

\author{
F. Bosch ${ }^{a, b}$, J.E. Baños ${ }^{a}$
}

Introducción. Tradicionalmente, las ciencias sociales y las experimentales han seguido caminos distintos, lo que ha llevado a la creación de una separación artificial entre ellas. Uno de los ejemplos más evidentes que han intentado evitar tal situación es la creación de disciplinas como la historia de la ciencia, que contribuye a acercar unas y otras. No obstante, la percepción entre los científicos del interés de la historia no es óptima a pesar de las indudables virtudes que contiene. Materiales y métodos. En el presente artículo describimos la experiencia de utilizar episodios históricos para contribuir al aprendizaje de procesos complejos, como es el de la investigación y desarrollo de nuevos medicamentos. Se describe el empleo de dos casos que culminaron con éxito y fueron galardonados con el premio Nobel: la modificación de la estructura química de neurotransmisores para obtener nuevos medicamentos (hormonas emasculadas) por James Black y la aplicación del concepto de antimetabolito para diseñar fármacos para el tratamiento de enfermedades infecciosas, neoplásicas y endocrinas. Conclusión. Tal aproximación docente es de notable éxito para hacer comprensible a los estudiantes las complejidades del proceso de investigación y desarrollo farmacéutico.

Palabras clave. Antimetabolito. Aprendizaje basado en problemas. Cimetidina. Farmacología. Historia de la ciencia. Investigación y desarrollo. Medicamentos. Propranolol.

Filling the gap: the usefulness of history of science to understand the process of drug research and development

Introduction. Traditionally, social and experimental sciences have followed different paths, which have led to the creation of an artificial separation between them. One of the clearest examples that has tried to avoid this situation is the creation of disciplines such as the history of science, which helps to bring both closer together. However, the perception among scientists regarding the interest of history is not optimal, despite the undoubted virtues that the latter contains. Materials and methods. In this article the experience of using historical events to contribute to learning of complex processes, such as research and development of new drugs, is described. The use of two cases is outlined that were successfully completed and awarded the Nobel Prize: the modification of neurotransmitters' structure to obtain new drugs (emasculated hormones) by James Black, and the application of the antimetabolite concept to design drugs for infectious, cancer and endocrine diseases. Conclusion. A teaching approach is remarkably successful in helping students understand the complexities of research and drug development.

Key words. Antimetabolite. Cimetidine. Drugs. History of science. Pharmacology. Problem based learning. Propranolol. Research and development.

\section{Introducción}

Desde que Paul Ehrlich aplicó los principios de la teoría inmunológica al desarrollo de nuevos fármacos [1], el descubrimiento de éstos dejó de constituir una mera aplicación del método ensayo-error para empezar a convertirse en una ciencia más o menos racional basada a partes iguales en la intuición, el trabajo sistemático y la suerte. A pesar de que el Salvarsan (como se conocía a la arsfenamina) constituyó un notable éxito conceptual, no lo fue tanto en la práctica y los médicos no dejaron de utilizar el paracelsiano mercurio hasta el descubrimiento de la penicilina en la década de

\footnotetext{
Departamento de Ciencias Experimentales y de la Salud. Facultad de Ciencias de la Salud y de la Vida. Universitat Pompeu Fabra.

b Fundación Dr. Antonio Esteve. Barcelona, España.

Correspondencia Dr. Josep Eladi Baños. Departamento de Ciencias Experimentales y de la Salud. Universitat Pompeu Fabra. Parc de Recerca Biomèdica de Barcelona. Doctor Aiguader, 88. E-08003 Barcelona.

Fax +34933160901.

E-mail

josepeladi.banos@upf.edu

Agradecimientos Los autores agradecen la ayuda y valiosos consejos del Prof. Luis A. Branda en la preparación del texto y objetivos del caso'Una carrera contaminada y sin doctorado'.
} 
los años treinta del pasado siglo [2]. En los años siguientes se descubrieron numerosos fármacos que cambiaron la concepción de la medicina y permitieron abandonar el nihilismo terapéutico practicado hasta entonces. Se acuñó incluso el término de 'revolución terapéutica' para referirse a la aparición de numerosos medicamentos en los aproximadamente veinte años que van desde la publicación del artículo de Florey y Chain demostrando la eficacia de la penicilina a la descripción de la focomelia causada por talidomida [3]. De alguna manera, los efectos teratogénicos causados por ésta despertaron a los científicos de un sueño: la obtención de medicamentos eficaces y seguros de forma ilimitada.

Existe abundante bibliografía que ilustra de forma detallada los descubrimientos más importantes de esta época [4-6]. Su análisis es especialmente útil para conocer las incertidumbres asociadas a la investigación de nuevos medicamentos, los problemas asociados a su desarrollo y la génesis de los paradigmas que permitieron el avance científico en el ámbito de la farmacología, el proceso que conduce a probar su validez y, finalmente, todas las consecuencias derivadas de su éxito o fracaso.

En el presente artículo se exponen dos ejemplos de cómo la historia de la ciencia puede contribuir en el ámbito docente a comprender el complejo mundo de la investigación científica en el campo del descubrimiento de nuevos medicamentos. Se expone la experiencia realizada en estudiantes de biología de la Universitat Pompeu Fabra con el empleo de la metodología docente del aprendizaje basado en problemas (ABP) dentro del itinerario profesional de industria sanitaria [7].

\section{Materiales y métodos}

\section{Situaciones problemáticas}

Los dos casos utilizados como situación problemática en las sesiones de ABP fueron 'Del whisky al aquavit: un largo y productivo viaje' y 'Una carrera contaminada y sin doctorado'. El primero se refiere a los descubrimientos de Sir James Black que cristalizaron en la comercialización del propranolol y de la cimetidina [8]. El segundo se refiere al trabajo conjunto de Gertrude Ellion y George Hitchings, que permitió obtener fármacos de gran importancia clínica como la mercaptopurina, el aciclovir y el alopurinol [9]. Cada situación problemática constaba de dos partes, un primer texto y un segundo texto para su utilización en sesiones consecutivas.

\section{Metodología}

Los problemas se utilizaron con los alumnos de quinto curso de Biología de la Universitat Pompeu Fabra en el itinerario de industria sanitaria. Sus características académicas y docentes se han descrito previamente [7]. De forma breve, tras los cuatro primeros años comunes, los estudiantes escogen un itinerario optativo entre tres posibilidades: investigación biomédica, análisis clínicos e industria sanitaria. Este último se centra en ofrecer una especialización en el ámbito del desarrollo de nuevos fármacos a través de empresas del ámbito sanitario, especialmente empresas farmacéuticas. La formación se realiza en tres trimestres, el primero dedicado a proporcionar los conocimientos teóricos necesarios, y los dos siguientes, a la formación práctica mediante una estancia a dedicación completa en equipos de cada ámbito y a la realización de un trabajo de fin de licenciatura.

En el primer trimestre, los estudiantes del itinerario de industria sanitaria cursan dos asignaturas: 'Desarrollo de nuevos fármacos y productos sanitarios' y 'Farmacognosia y tecnología farmacéutica'. La metodología docente empleada consiste en clases magistrales, seminarios de casos y sesiones de ABP. Estas últimas se realizan en grupos de 8-12 estudiantes bajo la supervisión de un tutor. Fue en este contexto donde se emplearon los casos descritos en este artículo, en períodos de estudio que duraron tres semanas en cada caso y en los que los tutores se reunieron con los estudiantes en tres sesiones de dos horas de duración cada una. Siguiendo el esquema propuesto por Branda [10], en la primera sesión se analiza la primera parte de la situación problemática y se identifican los objetivos educativos. En la segunda sesión se presentan y debaten los hallazgos del grupo sobre los temas planteados en la primera, se presenta el texto correspondiente a la segunda parte del problema y se establecen los nuevos objetivos a alcanzar en la próxima sesión. En la tercera se resuelven todos los temas pendientes hasta dar por cerrado el problema. La secuencia de actividades que se realizan se resumen en la figura. 


\section{$1^{\text {a }}$ sesión de ABP \\ 1. Presentación $1^{\mathrm{a}}$ parte situación problemática \\ 2. Lluvia de ideas y creación de una lista de temas \\ 3. Confección del plan de aprendizaje \\ 4. Elección temas a desarrollar por los estudiantes}

\section{$2^{\mathrm{a}}$ sesión de ABP}

1. Presentación y debate sobre los temas seleccionados

2. Presentación $2^{\mathrm{a}}$ parte situación problemática

3. Lluvia de ideas y creación de una lista de temas

4. Nuevo plan de aprendizaje

5. Elección temas a desarrollar por los estudiantes

\section{$3^{\text {a }}$ sesión de ABP}

1. Presentación y debate sobre los temas seleccionados

2. Reevaluación del problema inicial

3. Evaluación conjunta de las tres sesiones

Figura. Diagrama de flujo de las actividades desarrolladas con el empleo de los textos.

\section{Evaluación}

Una vez finalizado el caso y durante la tercera sesión del ABP, los tutores pidieron a los estudiantes que completaran de forma anónima y voluntaria un cuestionario en el que se solicitaba información sobre el funcionamiento del caso, el grado de mejora de los conocimientos sobre los principales objetivos educativos que se habían preestablecido, la contribución del estudiante a la situación problemática, los aspectos que más y menos habían interesado, la dedicación temporal fuera del aula y las sugerencias de mejora de todo el proceso. Los resultados numéricos se calcularon como media e intervalos. Se obtuvieron ejemplos de las respuestas de los estudiantes en las preguntas abiertas.

\section{Resultados}

\section{Análisis del problema 'Del whisky al aquavit: un largo y productivo viaje'}

Las dos partes de este problema se presentan en las tablas I y II, respectivamente. Sus objetivos principales de aprendizaje se resumen en la tabla III. Como puede observarse, se pretendía presentar a los estudiantes el proceso de investigación y desarrollo de las dos principales contribuciones de Black mediante su paradigma experimental de la modificación de la estructura de neurotransmisores para que éstos actuaran como antagonistas funcionales de las sustancias endógenas. Asimismo, se pretendía que los estudiantes analizaran la 
Tabla I. Primera parte de ‘Del whisky al aquavit: un largo y productivo viaje’.

Después de los doce años transcurridos desde su graduación en la Universidad de Saint Andrews, la idea que le rondaba por la cabeza parecía difícil de realizar en cualquier universidad de su país. Había trabajado como académico durante todo este tiempo, pero ahora parecía que era el momento de cambiar y había decidido aceptar una oferta de la industria farmacéutica.

En la nueva empresa las cosas fueron bien desde el principio. La idea de que el dolor que sufrían los pacientes durante un ataque de angina de pecho se debía a la llegada de poco oxígeno al miocardio era aceptada en esa época y existían varias evidencias que apoyaban esta posibilidad. La cuestión era cómo mejorar a los pacientes mediante la administración de medicamentos. ¿Podrían modificar la fisiopatología de la enfermedad? Las teorías de Ahlquist, publicadas unos años atrás, sugerían la posibilidad de modular selectivamente la acción de los neurotransmisores del sistema nervioso vegetativo. La cuestión, en cualquier caso, era cómo hacerlo en el corazón.

Al mismo tiempo que consideraba su paso a la empresa farmacéutica, los investigadores de otro laboratorio habían demostrado que esta posibilidad era real con un nuevo fármaco que estudiaban para el tratamiento del asma. Sin embargo, cuando se analizó en detalle, mostró que tenía efectos que podían perjudicar el objetivo de encontrar un medicamento para la angina. Era, no obstante, un buen punto de partida ya que su modificación estructural permitió obtener un derivado que carecía de tales problemas. Parecía que se encontraban al final del camino con el ICI 38174 aunque, en realidad, la historia acababa de empezar.

Pronto los toxicólogos trajeron las malas noticias: el ICI 38174 era tóxico en ratones y no era posible continuarlo en la fase clínica. No se desanimaron. Quizá una nueva modificación permitiría obtener un derivado sin tales problemas. Así fue: el nuevo fármaco mantenía las propiedades del anterior y estaba desprovisto de su toxicidad. Por fin lo habían encontrado.

\section{Tabla II. Segunda parte de ‘Del whisky al aquavit: un largo y productivo viaje’.}

El éxito del nuevo medicamento fue inmediato, tanto comercialmente como desde la óptica conceptual. Supuso una nueva posibilidad en la angina de pecho y en el tratamiento de la isquemia miocárdica en general. Pero él era un hombre inquieto y, cuando los estudios clínicos se iniciaron, emigró a una nueva empresa. El reto era ahora la histamina y la secreción gástrica. Por analogía con los estudios previos con los antagonistas de los $\beta$-adrenoceptores, se deseaba encontrar un medicamento para la úlcera gastroduodenal. Su contribución revolucionaría la terapéutica de esta enfermedad para siempre. La pequeña compañía de Filadelfia que había participado en su desarrollo y comercialización se convertiría en una gran empresa. Años después, sin embargo, los vientos soplarían en contra.

Mientras tanto, en su primera empresa, las cosas no podían ir mejor. El nuevo medicamento mostró su eficacia en una enfermedad de elevada prevalencia y además se describieron efectos beneficiosos en muchas situaciones clínicas en el ámbito de la neurología, la endocrinología e, incluso, en el tratamiento de enfermedades infecciosas. Alguien dijo, incluso, que debían probarlo en los pies planos. Este amplio abanico de indicaciones tenía mucho que ver con algunas de las propiedades del medicamento.

Pero la vida da muchas vueltas. Así, la empresa que comercializaba su invención primigenia pronto cambiaría de nombre y se fusionaría con otra que, curiosamente, fue la primera en comercializar el medicamento que supone una de las referencias actuales en el tratamiento de la úlcera gastroduodenal y que con el tiempo acabó desplazando al obtenido en la compañía de Filadelfia, una vez superaron unos problemas iniciales de toxicidad.

¿Y qué pintan los licores en esta historia? Para descubrirlo, deben consultarse las noticias que sucedieron en Estocolmo en el invierno de 1988

lógica aplicada por Black a sus descubrimientos y los obstáculos encontrados con las primeras moléculas hasta conseguir finalmente las que se utilizaron en terapéutica. En la segunda parte, los estudiantes debían conocer además algunos aspectos importantes del desarrollo de nuevos medicamentos, como las fusiones de empresas farmacéuticas o el desplazamiento de los productos líderes por nuevos fármacos basados en el desarrollo de conocimientos sobre la etiología de las enfermedades.

\section{Evaluación}

La tabla VII (caso 1) recoge los resultados de la 
Tabla III. Objetivos educativos específicos del problema 'Del whisky al aquavit: un largo y productivo viaje'.

- Recordar la fisiopatología de la angina de pecho

- Comprender las bases de la terapéutica de la angina de pecho

- Conocer las fases de la investigación preclínica de medicamentos

- Recordar la fisiopatología de la úlcera gastroduodenal

- Analizar la importancia de los subtipos de adrenoceptores

- Revisar la neurotransmisión en el sistema nervioso vegetativo

- Comprender cómo la modificación de las estructuras químicas afecta a la eficacia y seguridad de los medicamentos

- Aprender el tratamiento actual de la úlcera gastroduodenal

- Conocer las razones de las fusiones de las empresas farmacéuticas

- Aprender la nomenclatura de los medicamentos

Tabla IV. Primera parte de ‘Una carrera contaminada y sin doctorado'.

Una mañana de octubre recibí una llamada telefónica inesperada. No me podía creer lo que el periodista me informaba sobre el reconocimiento que la comunidad científica me había concedido. En aquel mismo momento me pasaron por la cabeza infinidad de imágenes y recuerdos. De muy joven, cuando mi abuelo, al que quería mucho, murió de cáncer, decidí que ésta sería la enfermedad contra la que debería luchar. Tras varios años que fueron difíciles, en los cuales debí pagar mis estudios enseñando bioquímica y trabajando en laboratorios de control de calidad, pude integrarme en un grupo de trabajo que sintetizó un compuesto que la FDA aprobaría pocos años más tarde. Todo ello fue posible gracias a los estudios previos sobre el metabolismo de las purinas y pirimidinas. Los datos se completaron paso a paso y todos los esfuerzos hasta la evaluación del nuevo fármaco en enfermos estuvieron justificados.

Su empleo clínico presentaba, sin embargo, ciertos problemas que conllevarían la necesidad de profundizar en otras estructuras químicas más eficaces. Quedaban todavía muchas incógnitas por resolver sobre el metabolismo del compuesto que requerirían ser analizadas con el paso del tiempo.

Entre las reflexiones revividas tras la desconcertante llamada, recordé las complicaciones históricas que desde el punto de vista social, político y económico acontecieron durante mis inicios en la investigación. Personalmente, y a pesar de mi demostrada competencia, tuve que afrontar grandes retos y salvar obstáculos no siempre visibles y muchas veces no socialmente aceptados.

evaluación realizada por los estudiantes del curso académico 2008-2009 que utilizaron esta situación problemática. El $91 \%$ de los participantes eran mujeres con una mediana de edad de 22 años. Los resultados muestran que los estudiantes mejoraron sensiblemente su conocimiento sobre el proceso de investigación y desarrollo de nuevos medicamentos y valoraron positivamente la experiencia.

\section{Análisis del problema ‘Una carrera contaminada y sin doctorado'}

Las tablas IV y V recogen las dos partes del problema, mientras que los objetivos educativos específicos se resumen en la tabla VI. Este texto se dirige también a analizar el proceso de investigación y desarrollo, ahora desde otro ejemplo histórico como es la obra científica de Gertrude Elion realizada en colaboración con George Hitchings. Ambos investigadores desarrollaron el concepto de antimetabolito como estrategia terapéutica para diseñar nuevos fármacos que interfirieran con vías metabólicas de microorganismos, células neoplásicas o células normales. En el curso de la actividad, los estudiantes analizaron cómo el principio de interferencia por antimetabolitos puede aplicarse a diversas enfermedades, como el cáncer, la prevención del rechazo tras un trasplante, las enfermedades víricas o la hiperu- 
Tabla V. Segunda parte de 'Una carrera contaminada y sin doctorado'.

Los estudios iniciados por el grupo de Hitchings sobre la inhibición del Lactobacillus casei con diaminopurina culminaron inicialmente con la obtención de dos compuestos, uno de ellos el purinetol, comercializado en comprimidos después de demostrar su eficacia en el tratamiento de la leucemia. Estos primeros avances revolucionarían el campo de la quimioterapia antineoplásica y supondrían nuevas vías de investigación farmacológica. Se llevaron a cabo estudios que habían demostrado que el purinetol también podía bloquear la formación de anticuerpos en conejos, lo que permitió abrir un nuevo abordaje terapéutico para un profármaco que, tras demostrar su eficacia en fases clínicas, constituiría la clave en la introducción, años más tarde, de una nueva generación de herramientas terapéuticas.

Tabla VI. Objetivos educativos específicos del problema ‘Una carrera contaminada y sin doctorado'.

- Describir el papel de la investigación preclínica en el desarrollo de nuevos fármacos

- Describir las particularidades de los principales estudios (química, farmacología, toxicología, galénica) de la investigación preclínica

- Revisar la fisiopatología de diversas situaciones clínicas (leucemia, rechazo de trasplantes, infecciones herpéticas, hiperuricemia) para comprender el concepto de diana farmacológica

- Recordar la farmacología de los principales grupos farmacológicos abordados: antileucémicos (diaminopurina, 6MP), inmunosupresores (6MP, azatioprina), antivíricos (aciclovir) y antigotosos (alopurinol)

- Explicar con un ejemplo histórico de la investigación de medicamentos por qué ha tenido trascendencia en la terapéutica de la segunda parte del siglo xx

- Identificar las diferencias entre las estrategias de la investigación racional y de la investigación por 'ensayo y error' aplicados al mundo de los medicamentos

- Ser capaz de debatir sobre las presiones que rodean el mundo de la investigación farmacéutica (investigación con animales, bioética, factores económicos, factores políticos, condición de mujer como investigadora, fusiones empresariales, nuevas indicaciones de fármacos conocidos)

ricemia. Además, se analizaban las dificultades que sufrían las mujeres en la primera mitad del siglo $\mathrm{xx}$ para incorporarse al mundo de la investigación, situación ejemplificada por Gertrude Elion.

\section{Evaluación}

La tabla VII (caso 2) recoge los resultados de la evaluación realizada por los estudiantes del curso académico 2009-2010 con el empleo de este caso. El 76\% de los participantes eran mujeres y su mediana de edad, de 22 años. Al igual como ocurrió con el primer problema, los resultados muestran que los estudiantes mejoraron sensiblemente su conocimiento sobre el proceso de investigación y desarrollo de nuevos medicamentos y también valoraron positivamente la experiencia.

\section{Discusión}

La utilización de los acontecimientos históricos para ilustrar la situación actual de la ciencia no es un recurso muy extendido, más allá de su empleo en situaciones específicas muy relacionadas con la historia de la ciencia [11]. Sin embargo, no es retórico afirmar que resulta frecuente que las mismas situaciones se repitan con cierta asiduidad, especialmente cuando el tiempo transcurrido no es importante. Por ello, se puede aprender de lo ocurrido aunque sólo sea para que no vuelva a ocurrir.

En el presente artículo presentamos dos ejemplos de investigación basada en nuevos paradigmas que finalizó con un éxito importante en el campo de la terapéutica humana. El descubrimiento del propranolol y de la cimetidina por 
Tabla VII. Resultados de la evaluación por los estudiantes.

\begin{tabular}{lcccc}
\hline & \multicolumn{2}{c}{ Caso 1 } & \multicolumn{2}{c}{ Caso 2 } \\
& ('Del whisky al aquavit') & \multicolumn{2}{c}{ ('Una carrera contaminada') } \\
\cline { 2 - 5 } & Media & Intervalo & Media & Intervalo \\
\hline Sesiones de tutoría & 8,6 & $7,0-9,0$ & 8,5 & $7,0-9,5$ \\
\hline Sesiones de grupo sin tutor & 7,3 & $6,0-9,0$ & 6,2 & $2,0-9,0$ \\
\hline Mejora de conocimientos & 7,5 & $5,0-10,0$ & 7,8 & $3,0-10,0$ \\
\hline Participación del estudiante & 7,7 & $6,0-10,0$ & 7,3 & $5,9-9,0$ \\
\hline Evaluación del tutor & 8,7 & $7,0-10,0$ & 8,8 & $8,0-10,0$ \\
\hline
\end{tabular}

Puntuaciones -entre 0 (peor) y 10 (mejor) como valores límite- asignadas por los estudiantes mediante un formulario individual y anónimo de evaluación cumplimentado al final de la tercera sesión de cada caso de ABP. Datos obtenidos de las promociones $2008-2009$ para el caso $1(n=21)$ y $2009-2010$ para el caso $2(n=17)^{\circ}$

James Black y la aplicación de la teoría de los antimetabolitos por Hitchings y Elion que permitió encontrar numerosos fármacos fueron recompensados con la concesión del premio Nobel de Fisiología y Medicina en 1988 [12]. La narración de cómo sucedió todo se ha recogido en las conferencias Nobel que se encuentran a libre disposición y que constituyen un buen material didáctico $[8,9]$. En nuestra opinión, su lectura, así como la de los artículos originales o revisiones sobre tales descubrimientos, ayuda de forma sustancial a conocer cómo funciona el proceso de descubrir en farmacología [13-15]. Esta posibilidad es especialmente importante para aquellos estudiantes que quieren dedicar su vida profesional a la investigación farmacológica. Nuestros resultados muestran que tales ejemplos pueden percibirse como realmente útiles por parte de los estudiantes.

Otro debate es el marco docente donde tales ejemplos pueden resultar más útiles. Consideramos que el empleo de estos textos debe realizarse en un ámbito que permita el trabajo autónomo de los estudiantes y el debate bajo la dirección de un tutor-facilitador que conozca claramente los objetivos que deben alcanzarse. En este sentido, el ABP constituye una opción excelente, aunque otros sistemas de trabajo cooperativo, como el método del caso, también serían útiles [16]. Las limitaciones del ABP en la docencia son de sobras conocidas [17], aunque desearíamos destacar las dificultades para utilizarla en cursos con muchos estudiantes, la falta de disponibilidad de tutores adecuadamente formados y la obtención de textos adecuados. Sin embargo, el Espacio Europeo de Educación Superior ofrece sustanciales ventajas para su aplicación, sobre todo cuando debe trabajarse en grupos reducidos y, especialmente, en el ámbito de los másters universitarios [18]. En este último caso, los grupos son necesariamente reducidos y el empleo del ABP o métodos similares es recomendable y posible.

Las dificultades de la experiencia que se describe en este artículo no han sido importantes. El formato del texto que conlleva una búsqueda casi detectivesca de los protagonistas y la necesidad de resolver las preguntas es, en general, bien recibida por los estudiantes. El único problema aparente es la dificultad del grupo para trabajar de forma coordinada fuera de las sesiones de tutoría. Para ello se designa un coordinador entre los estudiantes, aunque esta estrategia no siempre funciona. Otra alternativa es reservar espacio y tiempo dentro del horario académico para permitir tales reuniones sin tutor, aunque tal opción no es estimada por todos los grupos, que prefieren reunirse de forma espontánea. Tampoco hemos observado el 'efecto copia' por el cual los informes finales se pasan de un año a otro. Ello puede obedecer a dos razones: la primera, que los estudiantes abandonan la facultad en el curso siguiente; la segunda, el hecho 
de que en el ABP se prima el debate y el análisis, algo difícil de copiar, a diferencia de otro tipo de actividades donde se considera básicamente la respuesta correcta y no el proceso por el que se ha llegado a ella.

En conclusión, el empleo de ejemplos históricos para ilustrar la investigación y el desarrollo farmacéutico constituye una opción sencilla, económica y eficaz. Es probable que esta opción pueda también aplicarse al conocimiento y debate de otros aspectos de la medicina, como la bioética, la relación médico-paciente, la bioquímica e incluso la biología molecular. La historia de las ciencias biomédicas está repleta de hechos de singular interés para ayudar a los estudiantes a comprender cómo funcionan las cosas y permitir que lo descubran por sí mismos. Constituye adicionalmente un medio de incrementar la motivación por aprender, una condición nada desdeñable en profesiones que precisan de la formación continua para seguir desempeñándola de forma óptima.

\section{Bibliografía}

1. Bosch F, Rosich L. The contributions of Paul Ehrlich to pharmacology: a tribute on the occasion of the centenary of his Nobel prize. Pharmacology 2008; 82: 171-9.

2. Gensini GF, Conti AA, Lippi D. The contributions of Paul Ehrlich to infectious disease. J Infect 2007; 54: 221-4.

3. Weinshilboum RM. The therapeutic revolution. Clin Pharmacol Ther 1987; 42: 481-4.

4. Mann J. The elusive magic bullet. The search for the perfect drug. New York: Oxford University Press; 1999.

5. Weatherall M. In search of a cure. New York: Oxford University Press; 1999.
6. Sneader W. Drug discovery: a history. Chichester, UK: Wiley; 2005.

7. Aramburu J, Bosch F, Sentí M, Baños JE. Los itinerarios profesionales como método para mejorar la inserción profesional de los licenciados en biología: la experiencia de la Universitat Pompeu Fabra. Educ Med 2006; 9: 23-30.

8. Black J. Drugs from emasculated hormones: the principle of syntopic antagonism. URL: http://nobelprize.org/ nobel_prizes/medicine/laureates/1988/black-lecture. pdf. [04.02.2010].

9. Elion GB. The purine path to chemotherapy. URL: http:// nobelprize.org/nobel_prizes/medicine/laureates/1988/ elion-lecture.pdf. [04.02.2010].

10. Branda LA. El aprendizaje basado en problemas. De herejía artificial a res popularis. Educ Med 2009; 12: 11-23.

11. Miller L, Schweingruber H, Oliver R, Mayes J, Smith D. Teaching neuroscience through web adventures: adolescents reconstruct the history and science of opioids. Neuroscientist 2002; 8: 16-21.

12. Rajut N. The Nobel chronicle 1988; James Whyte Black (b. 1924), Gertrude Elion (1918-99) and George H. Hitchings (1905-98). Lancet 2000; 355: 1022.

13. Quirke V. Putting theory in practice: James Black, receptor theory and the development of beta blockers at ICI 1958-1978. Med Hist 2006; 50: 69-92.

14. Stapleton MP. Sir James Black and propranolol. The role of basic sciences in the history of cardiovascular pharmacology. Tex Heart Inst J 1997; 24: 336-42.

15. Koenig R. The legacy of great science: the work of Nobel laureate Gertrude Elion lives on. Oncologist 2006; 11: 961-5.

16. McKeachie AJ, Svinicki M. Teaching tips. Strategies, research, and theory for college and university teachers. Boston: Houghton Mifflin; 2006.

17. Jones RW. Problem-based learning: description, advantages, scenarios and facilitation. Anaesth Intensive Care 2006; 34: 485-8.

18. Benito A, Cruz A, eds. Nuevas claves para la docencia universitaria en el Espacio Europeo de Educación Superior. Madrid: Narcea; 2005. 